\title{
Solution-Processed Multilayered Polymer Solar Cells Designed by Layer-by-Layer Assembly of Poly( $p$-phenylenevinylene)s with Dimethylsulfoxide
}

\author{
Kohji Masuda, Yoshifumi Ikeda, Hideo Ohkita, ${ }^{*}$ \\ Michihiro Ogawa, Hiroaki Benten and Shinzaburo Ito \\ Department of Polymer Chemistry, Graduate School of Engineering, Kyoto University \\ Fax: 81-75-383-2617, e-mail: ohkita@photo.polym.kyoto-u.ac.jp
}

\begin{abstract}
Multilayered polymer solar cells were fabricated by a combination of spin-coating and layer-by-layer (LbL) deposition techniques. A light-harvesting layer was precisely designed by $\mathrm{LbL}$ assembly of poly(p-phenylenevinylene) (PPV) and an anionic PPV derivative, poly[5-methoxy-2-(3-sulfopropoxy)-1,4-phenylenevinylene] (MPS-PPV). The remaining hole-transporting and electron-transporting layers were prepared by the spin-coating method. In order to improve optical properties of the MPS-PPV LbL film, dimethylsulfoxide (DMSO) was added to an aqueous solution of MPS-PPV. As a result, the absorption of PPV/MPS-PPV LbL films increased at around $500 \mathrm{~nm}$ compared with that of the reference LbL films fabricated from an aqueous solution of MPS-PPV. The short-circuit current density of the multilayered polymer solar cell was improved by $>10 \%$ compared to the reference cell with PPV/MPS-PPV $\mathrm{LbL}$ layers fabricated from the aqueous solution. Furthermore, the external quantum efficiency at around $500 \mathrm{~nm}$ was also improved, suggesting the increase in the light-harvesting efficiency of the LbL layer fabricated from the water/DMSO mixed solution of MPS-PPV.

Key words: layer-by-layer deposition, poly(p-phenylenevinylene), dimethylsulfoxide, heterojunction, polymer solar cell
\end{abstract}

\section{INTRODUCTION}

Organic solar cells have been intensively studied because of their light-weight, flexibility, large-area and cost-effective production.[1-3] In organic solar cells, a coulombically bound electron and hole pair (exciton) is generated first after the photon absorption and can dissociate into free charged carriers only at the interface of donor and acceptor materials. Furthermore, the hopping length of each carrier such as exciton, hole, and electron, which play an important role in the photon-to-current energy conversion, is limited on a scale of nanometers. Therefore, it is crucial to design the photon active layer with nanometer precision.

We have designed and fabricated solution-processed multilayered polymer solar cells by combining the spin-coating and layer-by-layer (LbL) deposition techniques.[4-7] The LbL deposition technique is a simple and versatile method for fabricating multilayered ultrathin films with various functional materials. Recently we developed a triple-layered polymer solar cells with poly( $p$-phenylenevinylene) (PPV) conjugated polymer in the light-harvesting LbL layer whose thickness and interfacial nanostructures were carefully controlled with nanometer precision. The solar cell exhibited an internal quantum efficiency as high as $50 \%$, which is indicative of efficient photon-to-current conversion, and hence a power conversion efficiency of $0.26 \%$.[5] We furthermore improved the LbL-based photovoltaic cell by introducing an anionic PPV derivative (MPS-PPV) instead of an inert anionic polymer and obtained a slightly higher power conversion efficiency $(0.28 \%)$.[6] On the other hand, the optical properties of MPS-PPV have been reported to be improved by addition of surfactants, [8] polyelectrolytes,[9] dendrimers, [10,11] salts,[12] and organic solvents such as dimethylsulfoxide (DMSO) [12] to the original aqueous solution. Herein, we demonstrate that the light-harvesting efficiency of multilayered polymer solar cells with PPV/MPS-PPV LbL layers can be improved by using a water/DMSO mixed solution of MPS-PPV as an LbL deposition solution.

\section{EXPERIMENTAL}

\subsection{Materials}

Figure 1 shows the PPV derivatives used in this study. For the fabrication of LbL films, cationic and anionic polyelectrolyte solutions were prepared as follows: a cationic PPV precursor, poly $(p$-xylene tetrahydrothiophenium chloride) (pre-PPV, Aldrich, $0.25 \mathrm{wt} \%$ ), aqueous solution was diluted to $1 \mathrm{mM}$ at $\mathrm{pH} 8-9$ with water and an anionic PPV derivative, poly[5-methoxy2-(3-sulfopropoxy)-1,4-phenylenevinylene] (MPS-PPV, Aldrich, $0.25 \mathrm{wt} \%$ ), aqueous solution was diluted to give a $2 \mathrm{mM}$ aqueous solution $(\mathrm{pH} 7)$ or a $2 \mathrm{mM}$ water/DMSO (1: 1 by volume) mixed solution ( $\mathrm{pH} 9$ ). The MPS-PPV solution was ultrasonicated for $2 \mathrm{~min}$ and filtrated through a $0.45-\mu \mathrm{m}$ membrane filter. For the fabrication of spin-coat films, an aqueous solution of poly(3,4-ethylenedioxythiophene) : poly(4-styrenesulfonate) (PEDOT:PSS, Aldrich, $1.3 \mathrm{wt} \%$ dispersion in $\mathrm{H}_{2} \mathrm{O}$, conductive grade) mixed with ethylene glycol and a blend solution of $o$-dichlorobenzene with $\mathrm{C}_{60}$ (Frontier 

with Dimethylsulfoxide

(a)

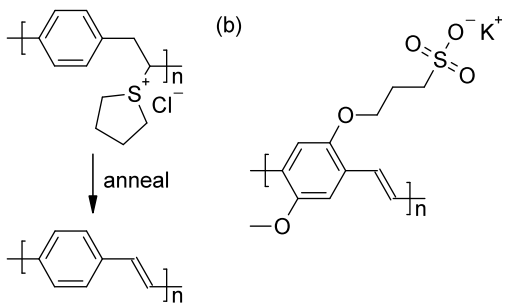

Fig. 1. Chemical structures of PPV derivatives employed in this study: (a) thermal conversion of pre-PPV to PPV, (b) MPS-PPV.

Carbon Co. Ltd.) and polystyrene (PS, Aldrich, $M_{\mathrm{W}}=$ 280,000) (4: 1 by weight) were used for a holetransporting and an electron-transporting layer, respectively.

\subsection{Device fabrication}

Indium-tin-oxide (ITO)-coated glass substrates $(10 \Omega$ per square) were cleaned by ultrasonication in toluene, acetone, and ethanol for $15 \mathrm{~min}$, respectively, and followed by surface treatment with a $\mathrm{UV}-\mathrm{O}_{3}$ cleaner for $60 \mathrm{~min}$. First, a hole-transporting layer was prepared on the pre-cleaned ITO substrate by spin-coating from a PEDOT:PSS solution, followed by thermal annealing at $70{ }^{\circ} \mathrm{C}$ for $12 \mathrm{~h}$ to give an insoluble film as reported previously.[7] Second, a light-harvesting layer was prepared by the LbL deposition of pre-PPV and MPS-PPV as follows. The PEDOT:PSS-coated ITO substrates were immersed in the pre-PPV solution for 5 min, and rinsed with pure water for $3 \mathrm{~min}$. Subsequently, they were immersed in the MPS-PPV solution for $5 \mathrm{~min}$ and rinsed with pure water for $3 \mathrm{~min}$. This cycle gives one-bilayer of pre-PPV/MPS-PPV LbL film. The LbL films were completely dried under a flow of air for 4-6 min after each immersion. Third, an electron-transporting layer was prepared on the LbL film by spin-coating from an $o$-dichlorobenzene solution of $\mathrm{C}_{60}$ and PS, followed by annealing at $100{ }^{\circ} \mathrm{C}$ for $2 \mathrm{~h}$ under vacuum to convert pre-PPV to PPV. Finally, Al was thermally deposited as a metal electrode on the top of the multilayered film through a metal mask to give an active area of $10 \mathrm{~mm}^{2}(2 \mathrm{~mm} \times 5 \mathrm{~mm})$. The layered structure of the multilayered solar cell is as follows: ITO|PEDOT:PSS $\mid(\text { PPV/MPS-PPV })_{n}\left|\mathrm{C}_{60}: \mathrm{PS}\right| \mathrm{Al}$.

\subsection{Measurements}

For the absorption and fluorescence measurements, LbL films were fabricated on quartz substrates. The UV-visible absorption spectra and emission spectra of PPV solutions and LbL films were measured with a spectrophotometer (Hitachi, U-3500) and a fluorescence spectrometer (Hitachi, F-4500), respectively. The thickness and the surface morphology of the films were measured by atomic force microscopy (AFM, Shimadzu, SPM-9500J) in the contact mode. The $J-V$ characteristics of the photovoltaic devices were measured with a DC voltage current source/monitor (Advantest, R6243). The photocurrent was measured under simulated AM1.5G solar illumination at $100 \mathrm{~mW}$ $\mathrm{cm}^{-2}$. The external quantum efficiency (EQE) spectra were measured with a digital electrometer (Advantest, R8252) under monochromatic light. This monochromatic light was obtained by passing white light from a 500-W Xe lamp (Thermo Oriel, Model 66921) through a monochromator (Thermo Oriel, UV-visible Conerstone) with optical cut-filters. All these measurements were performed in air at room temperature.

\section{RESULTS AND DISCUSSION}

First, we examined the optical properties of MPS-PPV in water and in a mixed solvent of water and DMSO. Figure 2 shows molar absorption coefficient and emission spectra of MPS-PPV in water (broken line) and in the mixed solvent (solid line). The absorption spectra were similar although the molar absorption coefficient was slightly larger in the mixed solvent. On the other hand, the emission intensity increased by a factor of six in the mixed solvent relative to that in water. These results are consistent with a previous report.[12] This is probably because DMSO is a better solvent for the polymer backbone and hence may reduce the interaction between the main chain. Therefore, MPS-PPV chains are likely to expand more in the mixed solvent.

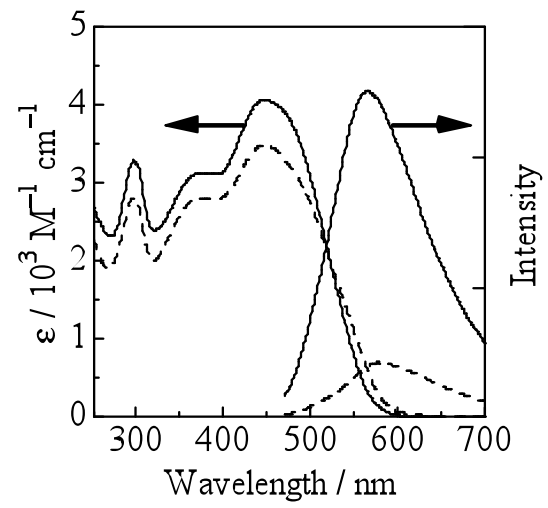

Fig. 2. Molar absorption coefficient and emission spectra of MPS-PPV in water (broken lines) and in the mixed solvent of water and DMSO (solid lines). The intensity was corrected for variations in the absorption at an excitation wavelength.

Next, we focus our attention on optical properties of LbL films with MPS-PPV. Figure 3a shows absorption spectra of pre-PPV/MPS-PPV films fabricated with the aqueous solution of MPS-PPV before and after annealing. Before the thermal annealing, the small absorption in the visible region was ascribed to MPS-PPV because pre-PPV has no absorption in that region. After the thermal annealing, a large absorption band was observed at around $400-500 \mathrm{~nm}$, which is ascribed to $\pi-\pi^{*}$ transitions of thermally converted PPV. As reported previously,[6] the small absorption of MPS-PPV may be due to a broad distribution of effective conjugation lengths. In contrast, as shown in Figure $3 \mathrm{~b}$, a distinct absorption band was observed at around $500 \mathrm{~nm}$ even before the thermal annealing. This band was safely ascribed to MPS-PPV because 


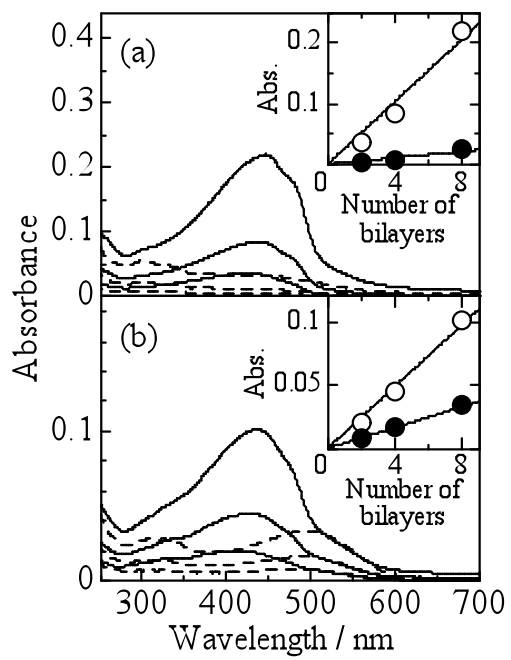

Fig. 3. Absorption spectra of PPV/MPS-PPV LbL films before (broken lines) and after (solid lines) annealing. The MPS-PPV layers were fabricated from a) the aqueous solution and b) the water/DMSO mixed solution. The insets of each figure show the absorption at $440 \mathrm{~nm}$ after annealing (open circles), at $500 \mathrm{~nm}$ before annealing (closed circles) of the PPV/MPS-PPV LbL films plotted against the number of bilayers.

there was no absorption due to pre-PPV. This is probably because MPS-PPV chains that are more expanded in the mixed solvent are likely to be adsorbed on the film surface in more extended conformations at the LbL deposition, which result in a longer absorption band. On the other hand, as shown in the insets of each figure, the absorbance at 440 and $500 \mathrm{~nm}$ increased proportional to the number of bilayers, indicating that the constant amount of pre-PPV and MPS-PPV is deposited at each LbL step.

To compare the film morphology, we measured AFM images of the PPV/MPS-PPV LbL films. For the reference LbL film with MPS-PPV fabricated from the aqueous solution, as shown in Figure 4a, the film surface was pinhole-free, uniform, and as smooth as the substrate surface, which is consistent with our previous report [6]. On the other hand, as shown in Figure 4b, the LbL film with MPS-PPV fabricated from the water/DMSO mixed solution was pinhole-free but not uniform: there were a lot of small bumps on the surface $\left(\sim 1.3 \mu \mathrm{m}^{-2}\right)$. A typical size of the bumps is $\sim 10 \mathrm{~nm}$ in height and $\sim 200 \mathrm{~nm}$ in diameter. From these results, the increase in the surface area due to the bumps is roughly estimated to be less than $1 \%$, and therefore negligible to the increase in the interface. Such bumps may be formed during immersion in an aqueous solution of pre-PPV, because water is a poorer solvent for the polymer backbone and may induce partial shrinking of extended MPS-PPV chains on the film surface. From the step as shown in the figure, each film thickness was measured and consequently the bilayer thickness was evaluated to be $3-4 \mathrm{~nm}$ for either LbL film.

We now move onto the device performance of multilayered polymer solar cells with PPV/MPS-PPV LbL layers. Figure 5 shows $J-V$ characteristics of the LbL-based solar cells with MPS-PPV fabricated from a)
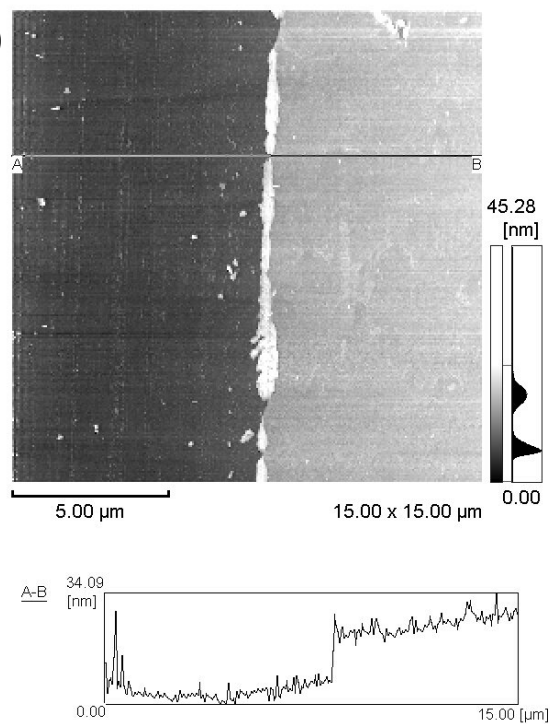

b)
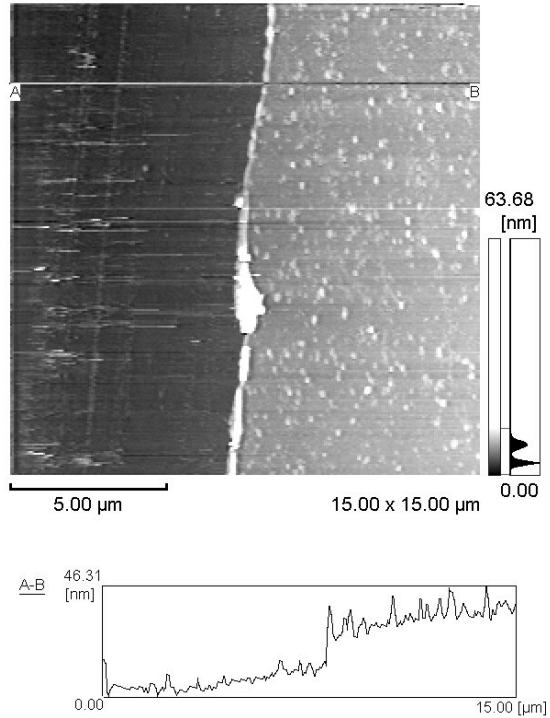

Fig. 4. AFM images of PPV/MPS-PPV LbL films with a) 6 bilayers and b) 8 bilayers. The MPS-PPV layers were fabricated from a) the aqueous solution and b) the water/DMSO mixed solution. The left side of each film was scratched out.

the water/DMSO mixed solution (solid line) and the reference cell from the aqueous solution (broken line). The thickness of the light-harvesting LbL layer was $\sim 10$ $\mathrm{nm}$, which is optimized for the power conversion efficiencies. For LbL-based solar cells with MPS-PPV fabricated from the water/DMSO mixed solution, the short-circuit current density $\left(J_{\mathrm{SC}}\right)$ was improved by $>$ $10 \%$ but the open-circuit voltage $\left(V_{\mathrm{OC}}\right)$ and the fill factor decreased and consequently the resulting power conversion efficiency was comparable relative to the reference cell. The decrease in $V_{\mathrm{OC}}$ suggests that the shunt resistance decreased because of the rougher surface of the PPV/MPS-PPV layer as shown in Figure $4 \mathrm{~b}$. On the other hand, the increase in $J_{\mathrm{SC}}$ suggests that the light-harvesting efficiency of PPV/MPS-PPV LbL layers is improved by the fabrication of the MPS-PPV layer from the water/DMSO mixed solution. This 

with Dimethylsulfoxide

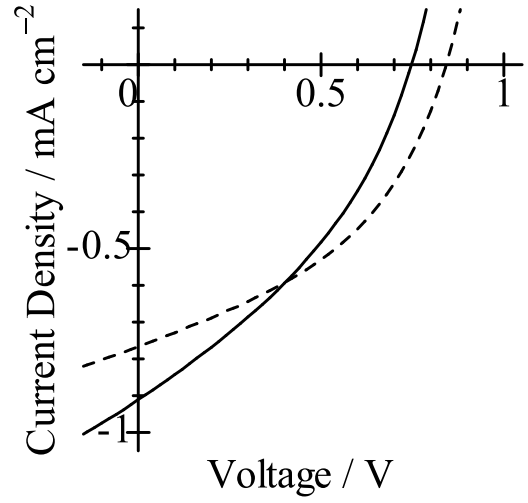

Fig. 5. $J-V$ characteristics of multilayered polymer solar cells with MPS-PPV fabricated from the aqueous solution (broken line) or the water/DMSO mixed solution (solid line) of MPS-PPV.

improvement is so large $(>10 \%)$ that it cannot be explained by the increase in the interface area due to the bumps on the surface $(<1 \%)$ as mentioned before. Therefore, it is rather ascribable to the absorption enhancement in the visible region at around $500 \mathrm{~nm}$ as shown in Figure 3. To confirm this we compared the EQE spectra of multilayered polymer solar cells with PPV/MPS-PPV LbL layers. As shown in Figure 6, a slight difference in the normalized EQE spectra was observed at around $500 \mathrm{~nm}$ : it was slightly larger in the cell fabricated from the water/DMSO mixed solution than in the reference cell. The slight increase in the absorption can account for an increase of $\sim 10 \%$ in $J_{\mathrm{SC}}$. The inset of the figure shows the spectral difference, which is consistent with the absorption of MPS-PPV layer as shown in Figure 3b. Therefore, we conclude that the fabrication of MPS-PPV layer from the water/DMSO mixed solution can enhance the absorption in the visible region and hence the light-harvesting efficiency of multilayered polymer solar cells.

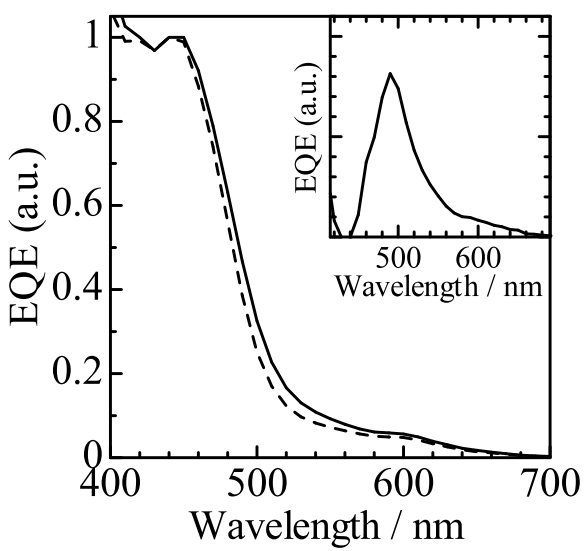

Fig. 6. Normalized EQE spectra of multilayered polymer solar cells with MPS-PPV fabricated from the aqueous solution (broken line) or the water/DMSO mixed solution (solid line). The inset shows the spectral difference between the two normalized EQE spectra.

\section{CONCLUSIONS}

We have shown that optical properties of LbL films with MPS-PPV conjugated polymer can be tuned by changing the LbL deposition solution. By using the water/DMSO mixed solution of MPS-PPV for the LbL deposition, the absorption at around $500 \mathrm{~nm}$ became more remarkable compared with the reference LbL film with MPS-PPV fabricated from the aqueous solution. As a result, $J_{\mathrm{SC}}$ of multilayered polymer solar cells was improved by $>10 \%$ relative to the reference cell fabricated from the aqueous solution. The increase in the EQE spectra at around $500 \mathrm{~nm}$ is consistent with the absorption enhancement and can account for the improvement in $J_{\mathrm{SC}}$. Therefore, we conclude that the light-harvesting efficiency of LbL-based multilayered solar cells can be improved by careful tuning of optical properties of LbL films.

\section{ACKNOWLEDGMENTS}

This work was partly supported by Kyoto University Global COE Program (International Center for Integrated Research and Advanced Education in Materials Science), Special Coordination Funds for Promoting Science and Technology from the Ministry of Education, Culture, Sports, Science, and Technology of Japan, by the Integrative Industry-Academia Partnership (IIAP) project including Kyoto University, Nippon Telegraph and Telephone Corporation, Pioneer Corporation, Hitachi, Ltd., Mitsubishi Chemical Corporation, and Rohm Co., Ltd., and by Takahashi Industrial and Economic Research Foundation.

\section{REFERENCES}

[1] S. Günes, H. Neugebauer, and N.S. Sariciftci, Chem. Rev., 107, 1324-1338 (2007).

[2] B.C. Thompson and M.J. Fréchet, Angew. Chem. Int. Ed., 47, 58-77 (2008).

[3] M. Jørgensen, K. Norrman, and F.C. Krebs, Sol. Energy Mater. Sol. Cells, 92, 686-714 (2008).

[4] H. Benten, N. Kudo, H. Ohkita, and S. Ito, Thin Solid Films, 517, 2016-2022 (2009).

[5] H. Benten, M. Ogawa, H. Ohkita, and S. Ito, $A d v$. Funct. Mater., 18, 1563-1572 (2008).

[6] M. Ogawa, M. Tamanoi, H. Ohkita, H. Benten, and S. Ito, Sol. Energy Mater. Sol. Cells, 93, 369-374 (2009).

[7] M. Ogawa, N. Kudo, H. Ohkita, S. Ito, and H. Benten, Appl. Phys. Lett., 90, 223107 (2007).

[8] J. Dalvi-Malhorta, and L. Chen, J. Phys. Chem. B, 109, 3873-3878 (2005).

[9] S. Abe, and L. Chen, J. Polym. Sci. Part B: Phys., 41, 1676-1679 (2003).

[10] M.S. Johal, M. Howland, J.M. Robinson, J.L. Casson, and H.L. Wang, Chem. Phys. Lett., 383, 276-281 (2004).

[11] G.A. Montaño, A.M. Dattelbaum, H.L. Wang, and A.P. Shreve, Chem. Commun., 2490-2491 (2004).

[12] A.D. Smith, C.K. Shen, S.T. Roberts, R. Helgeson, and B.J. Schwartz, Res. Chem. Intermed., 33, 125-142 (2007).

(Received May 19, 2009; Accepted November 17, 2009) 